\title{
Is the Tradition of Nasal Packing Just an Illusion of Permanence or a Necessary Evil?
}

\author{
Arulalan Mathialagan ${ }^{1}$ Suyash Singh ${ }^{2}$ Awadhesh Kumar Jaiswal² Ravi Sankar Manogaran ${ }^{1}$ \\ Prabhakar Mishra ${ }^{3}$ Amit Kumar Keshri² \\ ${ }^{1}$ Neuro-otology Unit, Department of Neurosurgery, Sanjay Gandhi \\ Post Graduate Institute of Medical Sciences, Lucknow, \\ Uttar Pradesh, India \\ 2Department of Neurosurgery, Sanjay Gandhi Post Graduate \\ Institute of Medical Sciences, Lucknow, Uttar Pradesh, India \\ ${ }^{3}$ Department of Biostatistics, Sanjay Gandhi Post Graduate Institute

\begin{abstract}
Address for correspondence Ravi Sankar Manogaran, MS, DNB, Neuro-otology Unit, Department of Neurosurgery, C-block, Sanjay Gandhi Post Graduate Institute of Medical Sciences, Raebareli Road, Lucknow, Uttar Pradesh 226014, India (e-mail: drravisankarpgi@gmail.com).
\end{abstract} of Medical Sciences, Lucknow, Uttar Pradesh, India

Indian J Neurosurg 2021;10:199-202.

\begin{abstract}
Background Nasal packing after an endoscopic endonasal approach (EEA) is a routine practice. There is a very urgent need for a level-I recommendation pertaining to the necessity of these packs and, if required, then the timing of its removal. However, the opponents of this practice mention its various disadvantages, among which infection is the most important. In our study, we have evaluated the bacteriological profile of the nasal packs, following endoscopic anterior skull base surgery.

Materials and Methods Thirty consecutive cases (2017-18) of anterior skull base pathologies operated by EEA were included, and preoperative nasal swab and postoperative period, the nasal packs were sent for microbiological culture. The colony of bacteria grew, and clinical condition, histopathology and demographic profile of the patients were noted. Results Of the 30 patients, $40 \%(n=12)$ showed an increase in nasal bacterial flora after packing, with methicillin-sensitive coagulase negative Streptococcus (MSCNS) in three patients, Escherichia fecalis (E. fecalis) in three patients, and methicillin-resistant coagulase negative Streptococcus (MRCNS) in two patients. Other rare organisms grew, including Acinetobacter baumanni with E. fecalis, Acinetobacter baumanni with MRCNS, Citrobacter koseni, and Escherichia fecium (E. fecium) with MRCNS and MRSA in one patient each.

Keywords

- anterior skull base surgery

- nasal packing

- bacterial culture

Conclusion Packing increases the bacterial load of the nasal cavity. This bacterial flora can be a potential source of meningitis. We advocate that packing should be avoided, and if at all required, should be removed within 3 days. A routine practice of nasal swab in the preoperative period and culture of the packs may give information on the possible organism that may cause meningitis and the appropriate antibiotic sensitivity of the organism.
\end{abstract}

\section{Introduction}

Endoscopic endonasal corridor has emerged as the first choice among all the approaches for pituitary adenoma, anterior skull base meningioma, majority of craniopharyngioma, and many other skull base tumors. One of the crucial

published online

March 11, 2021
DOI https://doi.org/ $10.1055 / s-0040-1716932$ ISSN 2277-954X. steps in the above approach involves reconstruction of skull base after tumor excision. Various techniques, including pedicle flap, reverse pedicle flap, mucosal flap, etc. have been described in literature. ${ }^{1}$ Nonetheless, use of nasal packs to reinforce the above reconstructions is one of the popular teachings practiced worldwide. However, as our insight 
in sinonasal physiology grew, the opponents of the nasal packing step pointed out its subclinical and unaddressed problems. One of the major issues related to the use of nasal packs is the possibility of infection or meningitis. This study aims to study the infective potential of nasal packs in postoperative period by analyzing bacterial growth over these packs.

The nasal cavity is known to harbor a natural nasal bacterial flora, and this cavity acts as gateway to anterior skull base and cranial fossa. Staphylococcus aureus is the commonest nasal commensal and the bacteria is implicated in many clinical infections. ${ }^{1}$ This fact was reported by Danbolt (1931) in his report on furunculosis. The methicillin-resistant Staphylococcus aureus (MRSA) is another potential pathogen implicated in wide range of infections. Majumdar et al reported that even the healthy population harbor a nasal carrier state of almost $25 \% .^{2}$ In a similar study by Kuehert et al, the Western population was studied, and Staphylococcus aureus and MRSA colonization was found to be present in nearly one-third of the population. ${ }^{3}$ These nasal carriers are supposed to have the maximum load and disperse organisms at the highest rate. ${ }^{4}$ These facts are important for skull base surgeons, as there is a theoretical possibility of introducing the above-mentioned nasal commensals into the cranial cavity during endoscopic endonasal surgery (EES). Henceforth, the practice of using nasal packing in EES became controversial, as it poses the potential risk of contracting meningitis. Despite being an important clinical issue, there is lack of substantial recommendation or large population-based studies guiding surgeons on the judicious use of nasal packing.

\section{Materials and Methods}

This is a prospective, observation, single institutional study, wherein the bacterial culture from the secretions of the post-EES nasal packs were analyzed. The study included the patients with central skull-based tumors irrespective of histopathology. Preoperative nasal swab was taken for aerobic culture to identify the normal nasal carrier state of the patient. The nasal packing was done with Merocel packs smeared with Betadine ointment bilaterally. The intention of using nasal packs was both to provide pressure over the nasal mucosa to achieve hemostasis and reinforce the skull base reconstruction. We removed the nasal packs between the 3rd and 5th postoperative day, and all the patients were administered empirical intravenous antibiotics for 48 hours. The pack removal was done in an aseptic manner and those packs were preserved in sterile containers. The containers were transferred immediately to our microbiology department. At the laboratory, the packs were squeezed, and the secretions were analyzed for aerobic and anaerobic culture in the BACTEC bottles. The growth was studied up to 72 hours of initial sampling. The data obtained was tabulated and analyzed using SPSS 22.0, IMB. Individual patient consent was taken to use clinical and radiological data for publication as per our departmental protocol.

\section{Statistical Analysis}

Continuous and normally distributed variable was represented as mean \pm standard deviation (SD) and categorical variable in frequency (\%). Association between two categorical variables were assessed using Fisher exact test. Simple bar diagrams and pie charts were used to present the categorical variables in terms of their frequency (\%). A two-tailed $p$ value $<0.05$ was considered as statistically significant. The statistical analysis was performed using the Statistical Package for Social Sciences, Version-23 (SPSS-23, IBM, and Chicago, USA

\section{Results}

Our study included 30 patients (M:F 15:15) with a mean age of 36.10 years ( $\mathrm{SD}=12.94$, range $9-61)$. Among these, $63.3 \%$ ( $n=19$ ) cases had pituitary macroadenoma, four cases had craniopharyngioma, and there was one case each of clival chordoma, olfactory grove meningioma, pituitary apoplexy, recurrent pituitary macroadenoma, right cavernous meningioma, right para clival mass and traumatic cerebrospinal fluid (CSF) rhinorrhea ( - Fig. 1). The preoperative nasal swab of two patients showed a positive smear. One patient had both $\mathrm{E}$. fecium and MRCNS, while the other patient tested positive for MRSA. The preoperative nasal swab of the rest of the 28 patients were found to be negative for organisms (-Fig. 2).

Among the postoperative nasal packs secretions culture, two patients who were nasal carriers (6.6\%) had the same organism in postoperative nasal pack secretions, while 12 patients (40\%) showed a new growth of organisms ( $p=0.6)$. The most common organism was MSCNS ( $n=3 ; 10.0 \%)$, followed by E. fecalis ( $n=3 ; 10.0 \%)$ MRCNS ( $n=2 ; 6.7 \%$ ), and Acinetobacter baumanni with E. fecalis, Acinetobacter baumanni with MRCNS, Citrobacter koseni, E. fecium with MRCNS and MRSA in one patient each (3.3\%). One patient showed MRCNS in anaerobic culture ( - Fig. 3 ). Of the patients in whom the pack was removed on and before 3 days, only three patients (out of $5,60 \%$ ) developed growth of organism

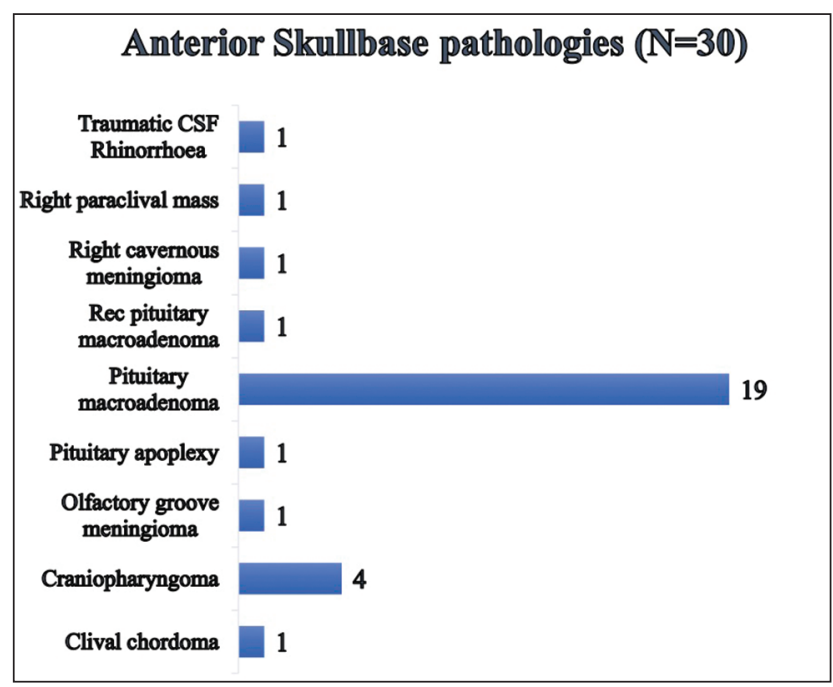

Fig. 1 Anterior skull base pathologies in the study group. 


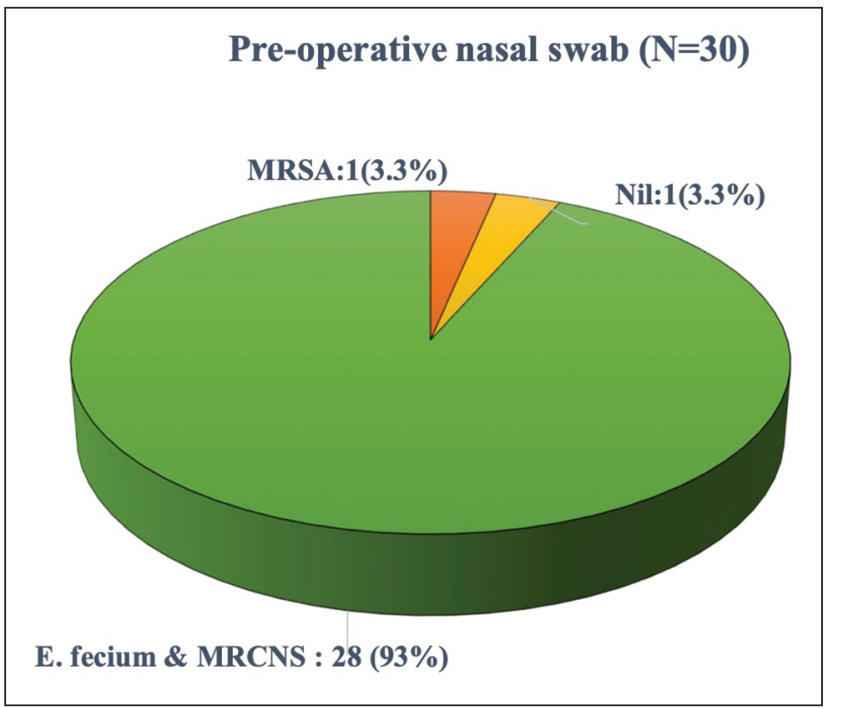

Fig. 2 Preoperative nasal swab in study patients. E. fecium, Escherichia fecium; MRSA, methicillin-resistant Staphylococcus aureus.

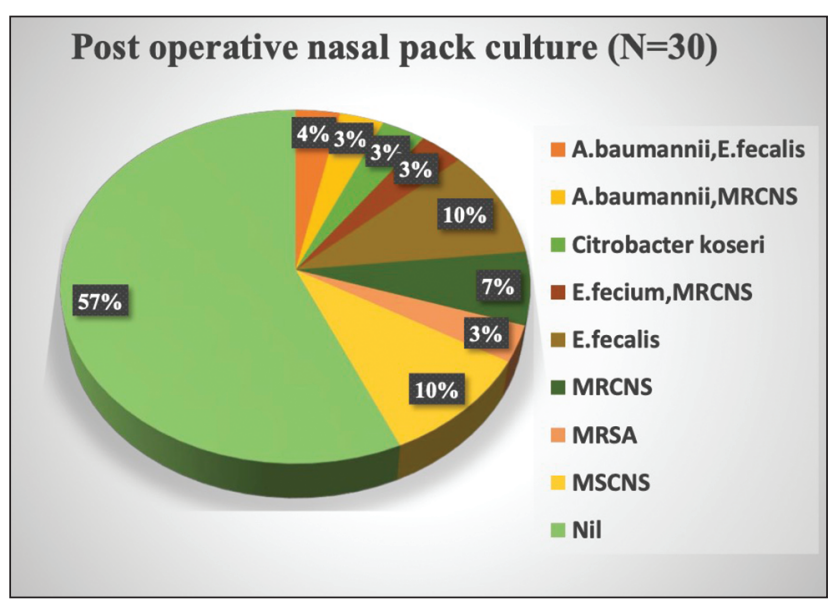

Fig. 3 Postoperative nasal culture in study patients. E. fecalis, Escherichia fecalis; E. fecium, Escherichia fecium; MRSA, methicillin-resistant Staphylococcus aureus; MSCNS, methicillin-sensitive coagulase negative Streptococcus; MRCNS, methicillin-resistant coagulase negative Streptococcus.

compared with 11 patients (out of $25,44 \%$ ) who developed growth of organism and among whom the nasal pack was removed on and after fourth day. Duration of packing did not show any statistically significant association with respect to development of new colonies of organism $(p=0.642)$.

\section{Discussion}

Although there are many synthetic materials available for the nasal packing, but cotton gauze smeared with antibiotic ointment is the most popular and conventional. The commercial nasal packs (Merocel) are expandable porous material made of polyvinyl acetate, while absorbable nasal packs made of esterified hyaluronic acid are also available. These nasal packs expand and exert pressure on the nasal mucosa and its blood vessels, thereby aiding in achieving hemostasis. In the last two decades, various authors focused on the complications and discomfort from nasal packs and argued against their use in skull base surgery.

In EES, these packs also support the skull base reconstruction. The nasal secretions tend to soak the porous nasal pack and provide an excellent culture media for bacteria. The nasal commensal can flourish in such a media, and pathogens can also grow in such a supple environment. Thus, the postoperative nasal cavity is loaded with commensals and pathogens, which are in close proximity to the skull base defect. Henceforth, the local transmission of bacteria and further meningitis are matters of concern. Several studies have evaluated meningitis after EES. Lai et al performed a systematic review of 42 articles and found an overall risk of $1.8 \%$ following EES. ${ }^{5}$ Further, some studies including patients with postoperative CSF leak showed much higher incidence (nearly $10-15 \%){ }^{6}$ Arbolay et al reported that not only did 20 to $30 \%$ of their cases develop meningitis but their series also registered a mortality of $20 \% .5,7,8$ In a similar study by Gupta et al, the pattern of bacterial growth in nasal packs used in packing epistaxis were analyzed, and they found that $86.6 \%$ nasal packs had bacterial colonisation. ${ }^{9}$ The quoted rate was much higher than ours, as they used loose cotton packs to pack the nasal cavity. Another study from Nepal found prevalence of bacterial growth to be $64.7 \% .^{10}$

In our study, we had packed the nasal cavity postoperatively, and the duration of retaining the nasal packs was based on two important factors, 1) hemostasis of the nasal mucosa at the end of surgery and 2) the size of anterior skull base defect repair. If there was minimal nasal mucosal bleeding at the end of the surgery and a small defect of the dura with a low-flow CSF leak, the nasal packs were removed in 2 days. In case of substantial nasal mucosal ooze or diffuse bleeding with a larger defect repair, the nasal packs were retained till 5 days.

In our study, the nasal bacteriological flora grew in $46.7 \%$ patients. Two of our patients, who were nasal carriers, showed same organism in the postoperative nasal pack culture also. Thus, $40 \%$ patients showed a nasal pack-induced change in the nasal flora. This proportion was similar in both the groups, that is, in the group where the packs were removed before 4 days and in second group, where pack was removed after that. Considering the small sample size, the statistical effect of duration of nasal packing with bacterial growth could not be ascertained. In spite of the fact that the nasal bacteriological flora changed in $40 \%$ of our patients, none of them developed meningitis. By performing the nasal swab preoperatively, we can ascertain the carrier state and hence get an idea of the culture and antibiotic assay. Therefore, if at all the patient develops meningitis in the postoperative period, we can start antibiotics accordingly.

\section{Conclusion}

Nasal packing increases the bacterial load in the nasal cavity, and these bacterial flora may be a potential source of meningitis. We advise to avoid these nasal packs, or if required, 
remove the packs within 48 hours. A larger randomized prospective population-based study is further needed to substantiate the conclusion. Our study is important in highlighting a critical step and lays the foundation for future research. A routine practice of nasal swab culture in the preoperative period and the culture on the packs can also provide information about the normal growth pattern and the selection of appropriate antibiotic assay.

\section{Conflict of Interest}

None declared.

\section{References}

1 Wertheim HFL, Melles DC, Vos MC, et al. The role of nasal carriage in Staphylococcus aureus infections. Lancet Infect Dis 2005;5(12):751-762

2 Majumdar D, Barua A, Paul B. Prevalence of nasal carriage of methicillin resistant staphylococci in healthy population of Gangtok, East Sikkim. J Int Med Sci Acad. 2008;21(4):191-193

3 Kuehnert MJ, Kruszon-Moran D, Hill HA, et al. Prevalence of Staphylococcus aureus nasal colonization in the United States, 2001-2002. J Infect Dis 2006;193(2):172-179
4 Maple PAC, Hamilton-Miller JMT, Brumfitt W. World-wide antibiotic resistance in methicillin-resistant Staphylococcus aureus. Lancet 1989;1(8637):537-540

5 Lai LT, Trooboff S, Morgan MK, Harvey RJ. The risk of meningitis following expanded endoscopic endonasal skull base surgery: a systematic review. J Neurol Surg B Skull Base 2014;75(1):18-26

6 Borg A, Kirkman MA, Choi D. Endoscopic endonasal anterior skull base surgery: a systematic review of complications during the past 65 years. World Neurosurg 2016;95:383-391

7 Arbolay OL, González JG, González RH, Gálvez YH. Extended endoscopic endonasal approach to the skull base. Minim Invasive Neurosurg 2009;52(3):114-118

8 Leong JL, Citardi MJ, Batra PS. Reconstruction of skull base defects after minimally invasive endoscopic resection of anterior skull base neoplasms. Am J Rhinol 2006;20(5):476-482

9 Gupta A, Agrawal SR, Sivarajan K, Gupta V. A microbiological study of anterior nasal packs in epistaxis. Indian J Otolaryngol Head Neck Surg 1999;51(1):42-46

10 Shrestha I, Pokharel M, Dhakal A, Amatya RCM, Madhup S, Sherchan JB. Study of microorganism growth pattern in nasal pack of patients visiting the Department of ENT, Head and Neck Surgery. Kathmandu Univ Med J (KUMJ) 2015;13(52):303-307 